\title{
Piercing oral: beleza, riscos e o papel da odontologia
}

\author{
Oral piercing: beauty, risks and the role of dentistry
}

Mariana Marquezan ${ }^{1}$, Leandro Teixeira Souza ${ }^{2}$, Orlando Tanaka ${ }^{3}$

\section{Resumo}

Piercings são adornos aplicados no corpo através de perfuração, e seu uso tem aumentado, em todo o mundo, entre jovens de diferentes classes sociais. $\mathrm{O}$ ato da aplicação da joia e até mesmo seu simples uso podem ser prejudiciais à saúde do paciente. As informações sobre riscos e malefícios do adorno, para que o paciente decida entre a moda e os possíveis prejuízos, devem ser fornecidas pelo cirurgião-dentista.

Palavras-chave: Piercing, Estética, Infecção, Complicações, Lesões

\section{Abstract}

Piercings are adornments applied to the body by means of perforations, and they are increasingly used among young persons of different social classes all over the world. The procedure to apply the ornament, or simply wearing it, can be harmful to the patient's health. The dentist must inform the patient about all the risks and harm resulting from the adornment, so that the patient can decide between following the fashion and the possible damage it may do.

Keywords: Piercing, Esthetics, Infection, Complications, Injuries

\section{Introdução}

A palavra piercing deriva do verbo inglês to pierce, que significa furar, perfurar, e é usada para definir um adorno de aço inoxidável, ouro, prata, teflon, acrílico ou titânio, fixado em diferentes partes do corpo, através de perfuração.

Há relatos de uso do piercing entre os egípcios, maias e nativos norte-americanos, tendo conotações espirituais, sexuais, estéticas e de rituais de passagem (BRENNAN; O'CONNELL; O'SULLIVAN, 2006). Na sociedade atual, piercings tornaram-se populares em todas as idades, ocupações e classes sociais (HUXLEY; GROGAN, 2005), sendo considerados uma forma de expressão, arte corporal (BRENNAN; O'CONNELL; O'SULLIVAN, 2006) ou simplesmente uma moda. Os primeiros relatos sobre piercing oral na literatura odontológica datam da década de 90 , sendo, portanto, um fenômeno relativamente novo para os dentistas (VENTÄ et al., 2005).

Não se tem estimativa de quantas pessoas usam piercing. Porém, Dubose e Pratt (2004) relataram que 17 a $51 \%$ de estudantes universitários têm piercing ou tatuagem nos Estados Unidos da América. Já na Finlândia, cerca de 3,4\% dos universitários entrevistados possuíam piercing oral, em 2002, (VENTÄ et al., 2005). Entre estudantes de Ensino Médio de Nova lorque, foi percebido que $10 \%$ possuíam piercing, sendo que mais da metade deles possuia entre 14 e 16 anos (PEAROSE; PERINPANAYAGAM; WELLS, 2006). Em estudo de Whittle (2006), a idade média dos indivíduos usuários de piercing foi de 19 anos.

$\mathrm{Na}$ cavidade bucal, os locais comumente utilizados são os lábios (Figura 1), a língua (Figura 2), o freio lingual (Figura 3) e o freio labial (Figura 4).
${ }^{1}$ Cirurgiã-Dentista graduada pela UFSM, aluna do Programa de Pós-Graduação em Odontologia, Área de concentração em Ortodontia - UFRJ.

${ }^{2}$ Mestre em Odontologia, Área de concentração em Ortodontia - PUCPR.

${ }^{3}$ Doutor em Ortodontia pela UFRJ e Professor Titular da Graduação e Pós-Graduação em Ortodontia - PUCPR

Correspondência: Mariana Marquezan

Endereço: R. Praia de Botafogo, 324, ap 504, Botafogo

CEP 22250-040 - Rio de Janeiro - RJ

Email: marianamarquezan@terra.com.br

Correspondência: Leandro Teixeira de Souza

Endereço: R. Antônio dos Reis Cavalheiro, 651/504, Cabral

CEP 80035210 - Curitiba - PR

Email: leandrotsouza@ terra.com.br

Correspondência: R. Orlando TanakaR Mal Deodoro, 630, 1703, Centro CEP 80010-912 - Curitiba - PR Email: tanaka.o@pucpr.br

Ao buscar, por estética ou moda, os usuários de piercings peri e intraorais estão sujeitos a riscos que muitas vezes desconhecem. Cabe ao cirurgião-dentista conhecê-los, para orientar seus pacientes.

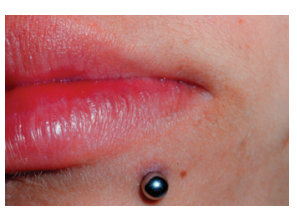

Figura 1. Piercing labial.
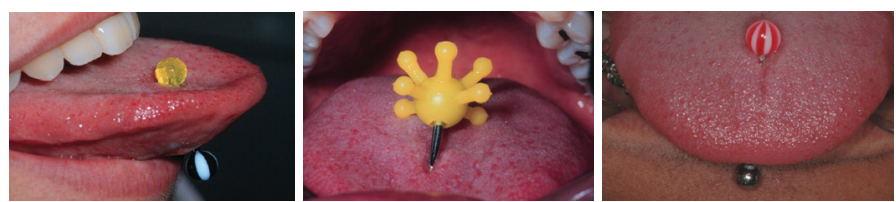

Figura 2. Diferentes modelos de piercing lingual.
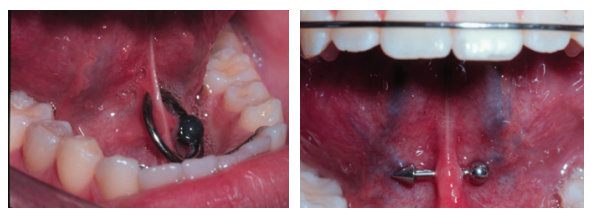

Figura 3. Casos de piercing no freio lingual.
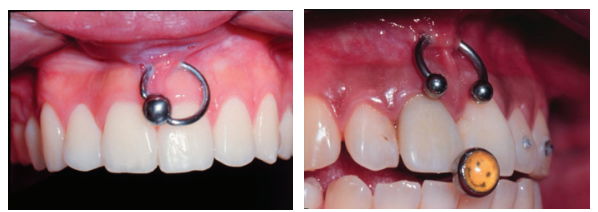

Figura 4. Casos de piercing no freio labial. 


\section{Revisão de literatura}

Os riscos oferecidos pelo uso de piercings orais são muitos. O primeiro deles pode ser devido ao ato de aplicação da joia, que, na maioria das vezes, não se dá por um profissional da saúde. Em estudo realizado por Kieser, Thomson, Koopu e Quick (2005), apenas 4 de 43 indivíduos entrevistados tiveram seus piercings aplicados por médicos ou dentistas na Nova Zelândia, os demais foram aplicados por colocadores de piercing ou piercers.

Piercers são pessoas sem habilitação profissional, autodidatas, que muitas vezes desconhecem a anatomia humana (SHACHAM et al., 2003), as condições sistêmicas do paciente e os parâmetros corretos de esterilização e assepsia (PEAROSE; PERINPANAYAGAM; WELLS, 2006), expondo o paciente, ou cliente, a doenças como tétano, hepatite, AIDS (AMERICAN DENTAL ASSOCIATION, 2001; SHACHAM et al., 2003; DUBOSE; PRATT, 2004; COSTA, 2004; LOPEZ-JORNET; CAMACHO-ALONSO, PONSFUSTER, 2005) e herpes (KIESER et al. 2005; LOPEZ-JORNET; CAMACHO-ALONSO; PONS-FUSTER, 2005). Na Inglaterra, porém, o estudo realizado por Whittle (2006) mostrou que a maioria dos piercers entrevistados se preocupa com infecção cruzada, utilizando inclusive autoclave. Entretanto, o conhecimento quanto a riscos de saúde associados ao piercing lingual foi variado.

Os colocadores de piercing não possuem licença para a utilização de anestésicos locais nem para prescrição de medicação pós-operatória, por isso, são comuns complicações como dor, edema (SHACHAM et al. 2003; HUXLEY; GROGAN, 2005; KIESER, 2005; LOPEZ-JORNET; CAMACHO-ALONSO; PONS-FUSTER, 2005) e hemorragias (AMERICAN DENTAL ASSOCIATION, 2001; LOPEZ-JORNET; CAMACHO-ALONSO; PONS-FUSTER, 2005).

Em estudo de Whittle (2006) 99\% dos indivíduos portadores de piercing relataram complicações, porém, apenas $7 \%$ deles procurou aconselhamento de um profissional da saúde. As complicações geralmente ocorrem de 4 a 6 semanas após a aplicação (SHACHAM et al. 2003). O edema decorrente da colocação de um piercing lingual, em casos extremos, poderá comprometer as vias aéreas superiores (SHACHAM et al. 2003). Se o piercing não for colocado exatamente sobre a linha média lingual, poderá causar sangramento prolongado e/ou parestesia, devido à presença de feixes vasculonervosos localizados paralelamente a ela (LOPEZ-JORNET; CAMACHO-ALONSO; PONS-FUSTER, 2005).

Além de complicações decorrentes da colocação do piercing, o uso da joia pode causar danos aos dentes, ao periodonto e aos tecidos de revestimento, tais como fratura dental (DE MOOR; DE WITTE; DE BRUYNE, 2000; AMERICAN DENTAL ASSOCIATION, 2001; CAMPBELL et al., 2002; LOPEZ-JORNET; CAMACHO-ALONSO; PONS-FUSTER, 2005; VENTÄ et al., 2005; BRENNAN; O'CONNELL; O'SULLIVAN, 2006; WHITTLE, 2006); trauma à mucosa (AMERICAN DENTAL ASSOCIATION, 2001; HUXLEY; GROGAN, 2005; LOPEZ-JORNET; CAMACHO-ALONSO; PONSFUSTER, 2005); recessão gengival associada a defeito ósseo periodontal (KRETCHMER; MORIARTY, 2001; CAMPBELL et al., 2002; BROOKS; HOOPER; REYNOLDS, 2003; CHOE; ALMAS; SCHOOR, 2005; KIESER, 2005; SOILEAU, 2005; LOPEZ-JORNET; CAMACHO-ALONSO; PONS-FUSTER, 2005; VENTÄ et al., 2005; LEICHTER; MONTEITH, 2006). As chances de se observar tais problemas na cavidade bucal aumentam com o tempo de uso do piercing (VENTÄ et al., 2005).

Há também relatos de: sensibilidade dental por galvanismo (DE MOOR; DE WITTE; DE BRUYNE, 2000; LOPEZ-JORNET; CAMACHO-ALONSO; PONS-FUSTER, 2005); reações alérgicas (AMERICAN DENTAL ASSOCIATION, 2001; HUXLEY; GROGAN, 2005; LOPEZ-JORNET; CAMACHO-ALONSO; PONS-FUSTER, 2005; VENTÄ et al., 2005); irritação na pele quando do uso de piercing labial, possivelmente por extravasamento de saliva (VENTÄ et al., 2005); halitose (LOPEZ-JORNET; CAMACHO-ALONSO; PONS-FUSTER, 2005); endocardite bacteriana (AMERICAN DENTAL ASSOCIATION, 2001; DUBOSE; PRATT, 2004; LOPEZ-
JORNET; CAMACHO-ALONSO; PONS-FUSTER, 2005); aspiração da joia (AMERICAN DENTAL ASSOCIATION, 2001; LOPEZ-JORNET; CAMACHO-ALONSO; PONS-FUSTER, 2005); ingestão da joia (WHITTLE, 2006); embolia cerebral (DUBOSE; PRATT, 2004) e infecções locais (AMERICAN DENTAL ASSOCIATION, 2001; KIESER, 2005; ANTOSZEWSKI et al., 2006). Infecções não tratadas podem evoluir para Angina de Ludwig (SHACHAM et al., 2003; KIESER, 2005; LOPEZ-JORNET; CAMACHO-ALONSO; PONSFUSTER, 2005).

Os aspectos colaterais voltados para os problemas funcionais também podem ocorrer, como dificuldade na mastigação, deglutição e fonação (AMERICAN DENTAL ASSOCIATION, 2001; LOPEZ-JORNET; CAMACHO-ALONSO; PONS-FUSTER, 2005). O usuário de piercing também pode desenvolver hábitos parafuncionais por brincar com a jóia, o que acentua os riscos de traumatismo e fraturas dentárias, além de poder gerar hiperatividade muscular.

Vistas tamanhas possibilidades de complicações, pacientes orientados sobre os riscos e malefícios do piercing que insistirem em utilizá-lo devem ser acompanhados pelo dentista (VENTÄ et al., 2005). A higiene da joia deve ser incentivada e até mesmo ser realizada pelo profissional, se necessário, visto que no estudo de Pearose, Perinpanayagam e Wells (2006), em que foram aplicados questionários a estudantes de Ensino Médio, cerca de $50 \%$ deles realizava a higiene da peça mensalmente e $21 \%$ anualmente.

Até mesmo o trabalho do dentista pode ser dificultado pelo adorno, pois joias aplicadas na região peri e intraoral podem prejudicar o diagnóstico de patologias, à medida que interferem nas imagens radiográficas (AMERICAN DENTAL ASSOCIATION, 2001; LOPEZ-JORNET; CAMACHO-ALONSO; PONS-FUSTER, 2005).

Quando da remoção do piercing, mais problemas podem surgir: cicatrizes podem comprometer a estética, especialmente na região labial (VENTÄ et al., 2005; ANTOSZEWSKI et al., 2006).

$\mathrm{Na}$ tentativa de proteger os menores de idade de todos esses riscos citados, a Associação Americana de Odontologia (American Dental Association-ADA) e a Academia Americana de Odontopediatria, nos Estados Unidos, são contra a aplicação de piercings intra e periorais nesses pacientes (DUBOSE; PRATT, 2004). No Estado de São Paulo, a lei $9.828 / 97$ proíbe que adolescentes com menos de 18 anos façam tatuagens e coloquem piercings. A Câmara de Vereadores de Curitiba, Paraná, em setembro de 2001, aprovou o projeto de lei 05.00019/2001, que permite ao menor realizar tatuagens e aplicar piercings, desde que haja consentimento dos pais ou responsáveis. Na Câmara dos Deputados Federais, tramitou, em 2001, o projeto de lei PL-5975/2001 que, em semelhança à lei paulista, proibiria aos menores o uso de piercings e tatuagens. Entretanto, esse foi arquivado, sendo aprovado o PL-1030/2003, que, em semelhança à lei curitibana, permite ao menor de 18 anos realizar tatuagens ou colocar adereços no corpo com autorização dos pais ou responsáveis.

Proibir o uso/aplicação de piercings e tatuagens por menores de idade sem o consentimento dos pais, entretanto, não é o bastante. Deve-se regulamentar a prática de aplicação, a fim de proteger todos os usuários, independentemente da idade. $\mathrm{Na}$ Europa, a Comissão Europeia (CE) quer que os Estados-Membros produzam legislação específica sobre piercings e tatuagens, de modo a minimizar os riscos para a saúde. Existem recomendações da União Europeia desde 2003, mas, dois anos depois, alguns países como Portugal continuam sem qualquer lei que regule ou permita fiscalizar a atividade (JESUS, 2005). No Brasil, alguns estados e municípios já apresentam leis para regulamentar e fiscalizar os estabelecimentos de aplicação de piercings e tatuagens, como a lei $\mathrm{n}^{\circ} 1337 / 1992$ do Mato Grosso do Sul, e a Lei Municipal n $10.169 / 2007$ de Porto Alegre, Rio Grande do Sul. 


\section{Discussão}

Não se tem estimativa de quantas pessoas façam uso de piercings intra e periorais, mas percebe-se um crescente aumento desse grupo de pacientes nos consultórios dentários. Sendo que os profissionais habilitados a intervir na cavidade bucal, os cirurgiões-dentistas, devem alertar seus pacientes sobre os riscos e possíveis malefícios inerentes à aplicação e ao uso. Deve-se salientar aos pacientes que os piercers não são profissionais capazes de oferecer nem conforto, nem segurança ao seu cliente, pois não poderão utilizar/prescrever anestésicos e medicamentos, por não terem formação em anatomia/fisiologia humana e biossegurança. Caso o paciente insista em aplicar um piercing, esse deve procurar um dentista ou médico, previamente para avaliação de sua saúde e preparo para receber a joia, o que pode incluir desde aconselhamentos à profilaxia antibiótica. A consulta com um profissional da saúde será também de valia para o controle dos sintomas pós-operatórios.

Em se tratando de paciente já usuário de piercing oral, o cirurgião-dentista deve orientá-lo quanto à higienização, pois o piercing, tendo uma superfície não descamável, é também capaz de acumular placa e cálculo dental. Instruções quanto à prática de esportes também devem ser dadas. Qualquer joia corporal deve ser removida durante os esportes, inclusive os piercings intra e periorais.

Estudos longitudinais deveriam ser realizados a fim de se observar os riscos e malefícios do uso de piercings orais, visto que a maior parte da literatura se trata de relatos de caso.

Seria interessante que, a exemplo da cidade de Porto Alegre, Rio Grande do Sul, e do estado do Mato Grosso, uma lei federal previsse a regulamentação e fiscalização dos estabelecimentos de aplicação de piercings e tatuagens. Enquanto não se institui uma sólida política nacional, cabe aos cirurgiões-dentistas exigir dos órgãos competentes medidas a fim de preservar a saúde dos pacientes, como campanhas de informação e a exigência de habilitação para a realização dessa prática.

Uma medida mais simples ainda que pode ser tomada pelos dentistas é o aconselhamento dos pacientes pela substituição do piercing bucal pelo piercing dental. Esse pode ser um cristal ou uma joia de ouro ou pedra preciosa, fixado no dente por meio de sistemas adesivos e resina composta, o que não danifica o esmalte dental nem necessita uso de anestésico, por não gerar dor, pode ser removido sem nenhum dano ao dente, quando não houver mais interesse, e não oferece riscos à saúde do paciente.

\section{Conclusão}

Tendo em vista o aumento crescente do uso de piercing oral entre pacientes jovens de diferentes classes sociais e conhecendo os riscos e malefícios desse adorno, cabe aos Cirurgiões-Dentistas orientar os pacientes quanto às desvantagens do uso e aos cuidados com a higienização e à prática de esportes, além de exigir sólida regulamentação e fiscalização dos estabelecimentos de body piercers pelos órgãos competentes.

\section{Referências}

AMERICAN DENTAL ASSOCIATION (ADA). Oral Piercing and Health. J. Am. Dent. Assoc., Chicago, v. 132, n. 1, p. 127, Jan. 2001.

ANTOSZEWSKI, B. et al. Are Body Piercing and Tattooing Safe Fashions? Eur. J. Dermatol., Mountrouge, v. 16, n. 5, p. 572-575, Sept./Oct. 2006.

BRENNAN, M.; O'CONNELL, B.; O'SULLIVAN, M. Multiple Dental Fractures Following Tongue Barbell Placement: a Case Report. Dent. Traumatol., Copenhagen, v. 22, no. 1, p. 41-43, Feb. 2006.

BROOKS, J. K.; HOOPER, K. A.; REYNOLDS, M. A. Formation of Mucogingival Defects Associated with Intraoral and Perioral Piercing: Case Reports. J. Am. Dent. Assoc., Chicago, v. 134, n. 7, p. 837-843, July 2003.

CAMPBELL, A. et al. Tongue Piercing: Impact of Time and Barbell Stem Length on Lingual Gingival Recession and Tooth Chipping. J. Periodontol., Indianapolis, v. 73, n. 2, p. 289-297, Mar. 2002.

CHOE, J.; ALMAS, K.; SCHOOR, R. Tongue Piercing as a Risk Factor to Periodontal Health. N.Y. State Dent. J., New York, v. 71, n. 5, p. 40-43, Aug./Sept. 2005

DE MOOR, R. J.; DE WITTE, A. M.; DE BRUYNE, M. A. Tongue Piercing and Associated Oral and Dental Complications. Endod. Dent. Traumatol., Copenhagen, v. 16, n. 5, p. 232-237, Oct. 2000.

DUBOSE, J.; PRATT, J. W. Victim of Fashion: Endocarditis After Oral Piercing. Curr. Surg., Philadelphia, v. 61, no. 5, p. 474-477, Sept./ Oct. 2004.

HUXLEY, C.; GROGAN, S. Tattooing, Piercing, Healthy Behaviours and Health Value. J. Health Psychol., London, v. 10, n. 6, p. 831-841, Dec. 2005

JESUS, S. Deco e UE Querem Lei Específica para 'Piercings' e Tatuagens. Diário de Notícias, Lisboa, 26 de maio 2005.

KIESER, J. A. et al. Oral Piercing and Oral Trauma in a New Zealand Sample. Dent. Traumatol., Copenhagen, v. 21, n. 5, p. 254-7, Oct. 2005.

KRETCHMER, M. C.; MORIARTY, J. D. Metal Piercing Through the Tongue and Localized Loss of Attachment: a Case Report. J. Periodontol., Indianapolis, v. 72, no. 6, p. 831-3, June 2001.

LEICHTER, J. W.; MONTEITH, B. D. Prevalence and Risk of Traumatic Gingival Recession Following Elective Lip Piercing. Dental Traumatol., Copenhagen, v.22, n. 1, p. 7-13, Feb. 2006.

LOPEZ-JORNET, P.; CAMACHO-ALONSO, F.; PONS-FUSTER, J. M. A Complication of Lingual Piercing: a Case Report. Oral Surg. Oral Med. Oral Pathol. Oral Radiol. Endod., St. Louis, v. 99, n. 2, p. 18-19, Feb. 2005

PEAROSE, M. M.; PERINPANAYAGAM, M. K.; WELLS, M. D. Trends in Oral Piercing in Buffalo, New York, High Schools. N.Y. State Dent. J., New York, v.72, n. 5, p. 30-32, Aug./Sept. 2006.

SHACHAM, R. et al. Tongue Piercing and its Adverse Effects. Oral Surg. Oral Med. Oral Pathol. Oral Radiol. Endod., St. Louis, v. 95, n. 3, p. 274-276, Mar. 2003.

Rev. Fac. Odontol. Porto Alegre., Porto Alegre, v. 49, n. 1, p. 12-15, jan./abr., 2008. 
SOILEAU, K. M. Treatment of a Mucogingival Defect Associated with Intraoral Piercing. J. Am. Dent. Assoc., Chicago, v. 136, n. 4, p. 490494, Apr. 2005.

VENTÄ, I. et al. Oral Piercings Among First-year University Students. Oral Surg. Oral Med. Oral Pathol. Oral Radiol. Endod., St. Louis, v. 99, n. 5, p. 546-549, May 2005.

WHITTLE, G. Investigating Tongue Piercing. Br. Dent J., London, v. 200, n. 2, p. 93, Jan. 2006. 